Relations industrielles

Industrial Relations

\title{
Cohn, Samuel, When Strikes Make Sense and Why: Lessons from Third Republic French Coal Miners
}

\section{Jacques Rojot}

Volume 50, numéro 2, 1995

URI : https://id.erudit.org/iderudit/051024ar

DOI : https://doi.org/10.7202/051024ar

Aller au sommaire du numéro

Éditeur(s)

Département des relations industrielles de l'Université Laval

ISSN

0034-379X (imprimé)

1703-8138 (numérique)

Découvrir la revue

Citer ce compte rendu

Rojot, J. (1995). Compte rendu de [Cohn, Samuel, When Strikes Make Sense and Why: Lessons from Third Republic French Coal Miners]. Relations industrielles / Industrial Relations, 50(2), 455-456. https://doi.org/10.7202/051024ar

Tous droits réservés (C) Département des relations industrielles de l'Université Laval, 1995
Ce document est protégé par la loi sur le droit d'auteur. L’utilisation des services d'Érudit (y compris la reproduction) est assujettie à sa politique d'utilisation que vous pouvez consulter en ligne.

https://apropos.erudit.org/fr/usagers/politique-dutilisation/ 
other when there is some interdependence between them; and for sustained relations to prevail, substantial interdependence is required. Although this interdependence can lead to conflict or cooperation, a crisis situation at the airline will likely bring forth more cooperation. The period under study saw increased cohesiveness among unions most likely reflected by the numerous crises faced by the industry. Nevertheless, even under a crisis situation union cooperation may not be complete as seen by the differences among the various unions on the question of the recent United Airlines buy-out. This is not necessarily surprising since mutual dependence is not the same as common interests. Given the fact that the situation in the airline industry will no doubt create more interdependence among the unions it is concluded that inter-union relations will continue at least at their current levels and possibly even increase.

To what extent can these conclusions concerning inter-union relations in the airline industry be generalized to other unions in other industries? This is an important industrial relations question because how unions relate to each other may also affect the outcome of the overall labor-management relationship. The author suggests further empirical research is necessary to answer this question. Nevertheless, one would expect that as other industries with multiunion representation find themselves in similar crisis situations, there will be an increase in inter-union relations. As these inter-union relations change one can expect the general overall labor-management relationship also to change. The specific manner in which this relationship changes remains to be seen.

For anyone interested in inter-union relations, labor relations in the airline industry, or indeed, organizational behavior in general, this book is well worth reading.

MARTIN M. PERLINE Wichita State University

\section{When Strikes Make Sense and Why: Lessons from Third Republic French Coal Miners}

by Samuel COHN, New York and London, Plenum Press, 1993, 254 p., ISBN 0306-44445-3.

Dans cet ouvrage, Cohn essaie de répondre à une question dont il constate, à juste titre, qu'elle a été fort peu analysée malgré son importance pour le champ des relations professionnelles: comment les stratégies des syndicats en matière de grève affectent-elles les salaires de ceux et celles qu'ils représentent ?

Pour ce faire, il utilise des données relatives aux mineurs de charbon français entre 1890 et 1935 . Elles présentent selon lui des avantages particuliers : fiabilité des statistiques salariales, bons éléments pour établir les déterminants du salaire non liés au conflit sur les salaires, excellentes statistiques de conflit social pour la période, stratégies divergentes des syndicats dans l'industrie (avec notamment, mais pas seulement, la scission de 1922 entre la CGT et la CGT-U), main-d'œuvre militante des mineurs. L'auteur applique ensuite des analyses de régression sur les séries temporelles (modèle de Parks) qu'il a recueillies et il double cette analyse quantitative d'une étude historique des sites sur la période.

Cette démarche l'amène à conclure que la stratégie syndicale optimale pour accroître les salaires est de déclencher des grèves fréquentes. Celles-ci donnent à un syndicat une réputation d'activisme qui, à son tour, impressionne les employeurs et les amène à conclure des accords plus généreux qu'ils ne seraient 
autrement. Toutefois, quand le mouvement syndical est divisé entre modérés et activistes (radicals au sens nordaméricain), cette stratégie est contreproductive car les employeurs réservent leurs concessions aux modérés qui font moins grève. Il est aussi notable que l'auteur considère que c'est le nombre de grèves qui est plus important que leur succès ou leur échec. Ce n'est pas de gagner une grève qui augmente les salaires. Les défaites peuvent être plus productives que les victoires, car les résultats acquis sont eux-mêmes relatifs au caractère important des revendications, qui génèrent une réputation d'activisme pour un syndicat.

Indiscutablement, l'idée est intéressante, et l'auteur expose clairement sa démarche. Cependant, l'ouvrage soulève plusieurs questions. D'une part, il se place dans une perspective théorique bien définie, ce qui amène à des simplifications que tous ne partageront pas. Il est, par exemple, un peu rapide d'affirmer en le tenant pour acquis au-delà de toute controverse que "le compromis de classe corporatiste abaisse les salaires " si il augmente le taux et la sécurité de l'emploi. Nombre de ceux qui ont étudié l'Allemagne et la Scandinavie peuvent avoir une opinion différente. Il est aussi simplificateur, et même un peu naïf, d'écrire que, alors que les salariés ne peuvent faire l'expérience de la grève et de son effet sur les salaires que tous les quatre ans (surtout en France !), les managers obtiennent des données quasi quotidiennes de l'effet sur les prix des volumes de ventes et des profits et qu'ils peuvent librement expérimenter en montant ou en descendant les prix pour en observer les effets. D'autres exemples pourraient être fournis tel l'utilisation du "pouvoir de classe" des mineurs, qui veut sans doute dire leur pouvoir de négociation, surtout quand il est basé, comme il l'est noté, sur l'appui de l'État, capitaliste en l'occurrence.

D'autre part, l'auteur, pour appliquer les techniques statistiques qu'il utilise, est naturellement amené à coder certaines de ses données. Les choix opérés alors, tels la décision de considérer un département comme "syndiqué " ou non, la correction des statistiques par les comptes-rendus de grèves, amènent, comme dans tous les cas d'études similaires, le lecteur à se demander si un résultat légèrement différent n'aurait pas été obtenu avec un codage lui aussi différent. Cependant, le fait que l'analyse statistique soit ensuite confortée par une analyse historique des sites concernés pallie dans une certaine mesure cet inconvénient. Le chapitre le moins convainquant est sans doute le dernier qui tente de généraliser les résultats de l'étude.

En résumé, l'ouvrage est intéressant et la thèse qu'il avance est provocante. Cependant, cette interprétation de l'exemple des mineurs de charbon français sous la troisième république suffit-elle à condamner les apports de l'école du Wisconsin aux relations professionnelles et à affirmer la supériorité générale du modèle de syndicalisme militant de conflit de classes en matière de salaires comme le fait son auteur? Le présent recenseur en doute fortement.

JACQUES ROJOT Université de Paris 1

\section{Strikes: Causes, Conduct and Consequences}

by Douglas BLACKMUR, Sydney, Federation Press, 1993, 232 p., ISBN 1-86287$114-0$

How powerful a tool is the mind? To what extent can the mind be harnessed to derive an understanding of real world phenomena? To understand the real world do we need to somehow or other become acquainted with literally every detail of that which becomes the object of our curiosity? Or is it possible to use 